\section{§2. Design of an Integrating Type Neutron Dose Monitor}

Yamanishi, H.

The radiation quality factors that ICRP recommends differ among neutron energies. In order to evaluate neutron dose precisely, it is expected that the neutron fluence of each energy group should be measured. REM counters are commonly used. However, these counters exhibit a large error for dose conversion in the intermediate energy range, since the fluence to dose conversion factor differs with the detector response, especially in this energy range. In several instruments developed prior to this study, multiple detectors were installed in a spherical polyethylene block. Those instruments are developed for measuring the energy spectrum and direction distribution of the neutron fluence. However, it is not suitable for radiation monitoring since the instrument needs complicated signal processing.

An integrating neutron dose monitor has been designed, which has lightweight and compact, good dose response, uniform sensitivity to all directions. In the instrument, the plural TLDs are arranged in the moderator at 3 levels of depth, in 12 directions. Although the TLDs were applied in this study, the other appropriate small detector may be adopted. The neutron dose can be measured correctly by the instrument with using three different energy response of the detectors. And the distribution of the value from the detectors may show a major incident direction of coming neutrons. The characteristic of the present monitor was discussed with the results of MCNP calculation.

The present designed instrument is spherical shape with $24 \mathrm{~cm}$ diameter, $9.1 \mathrm{~kg}$. Because of its shape, the sensitivity of the instrument is almost uniform whatever direction neutrons come from. TLD was selected as neutron detector in this study. The constitute parts of the TLD are $\mathrm{CaSO}_{4}$ and ${ }^{6} \mathrm{LiF}$, and the size is $12 \mathrm{~mm}$ long and 2 $\mathrm{mm}$ diameter. The moderator of the instrument is composed of spherical shell. Figure 1 shows the layer structure of the instrument. The detectors are arranged between layers of the moderators. The order of the arrangement from outside to the center is $20 \mathrm{~mm}$ thick polyethylene (PE), TLD1, $40 \mathrm{~mm}$ thick boron nitrite $(\mathrm{BN})$, TLD2, $45 \mathrm{~mm}$ thick PE and the center TLD3. The most outer TLD1 is for measuring neutrons of low energy, and TLD2 and TLD3 are sensitive to intermediate and high energy neutrons. The 12 sets of TLD1 and TLD2 are arranged 12 directions corresponding to the apexes of regular polyhedron with 20 sides. The total number of TLD is 25 . The response of the TLD, the reaction rate of ${ }^{6} \mathrm{Li}(\mathrm{n}, \alpha) \mathrm{T}$ was considered to be, was calculated using Monte Carlo calculation code MCNP-4B. The incident energies were chosen to less than $15 \mathrm{MeV}$.

The neutron dose is derived from the linear combination of 5 groups TLD value.

$$
\mathrm{D}=\mathrm{a}_{\mathrm{F} 1} \mathrm{~F} 1+\mathrm{a}_{\mathrm{F} 2} \mathrm{~F} 2+\mathrm{a}_{\mathrm{S} 1} \mathrm{~S} 1+\mathrm{a}_{\mathrm{S} 2} \mathrm{~S} 2+\mathrm{a}_{\mathrm{M}} \mathrm{M}
$$

$\mathrm{F} 1$ is the sum of the expected response of TLD1 in the 3 directions nearest to the neutron incident direction. $\mathrm{S} 1$ is the sum of the expected response of TLD1 in the 3 directions next to F-group. F2 and $\mathrm{S} 2$ correspond to TLD2. And $\mathrm{M}$ corresponds to the expected response of TLD3. The set of the dose conversion coefficient such as $\mathrm{a}_{\mathrm{F} 1}, \mathrm{a}_{\mathrm{F} 2}$ is selected to be good dose response by using the least-square method, comparing with the detector energy responses and the ideal dose curve. Figure 2 shows the calculated dose response from ${ }^{252} \mathrm{Cf}$ spontaneous fission source in the center of a $\mathrm{D}_{2} \mathrm{O}$ sphere with a radius of 150 $\mathrm{mm}$. The calculated dose response was well accorded in all energy range with the expected $\mathrm{H}^{*}(10)$ which was derived from the fluence. In addition, the direction of coming neutrons can be found out from the distribution of the measured value TLD1. And, the rough constitution ratio of low energy to intermediate energy can be obtained by the measured value of TLD1 and TLD2.

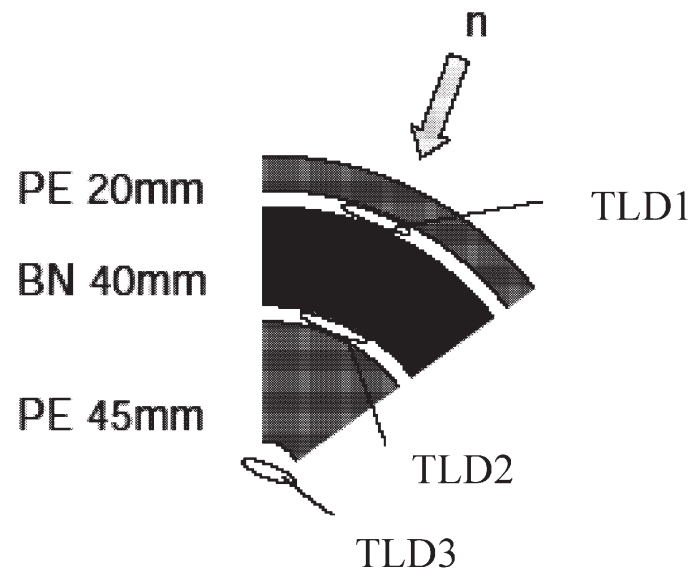

Fig. 1 Layer structure of the instrument. PE: Polyethylene, $\mathrm{BN}$ : Boron nitrate.

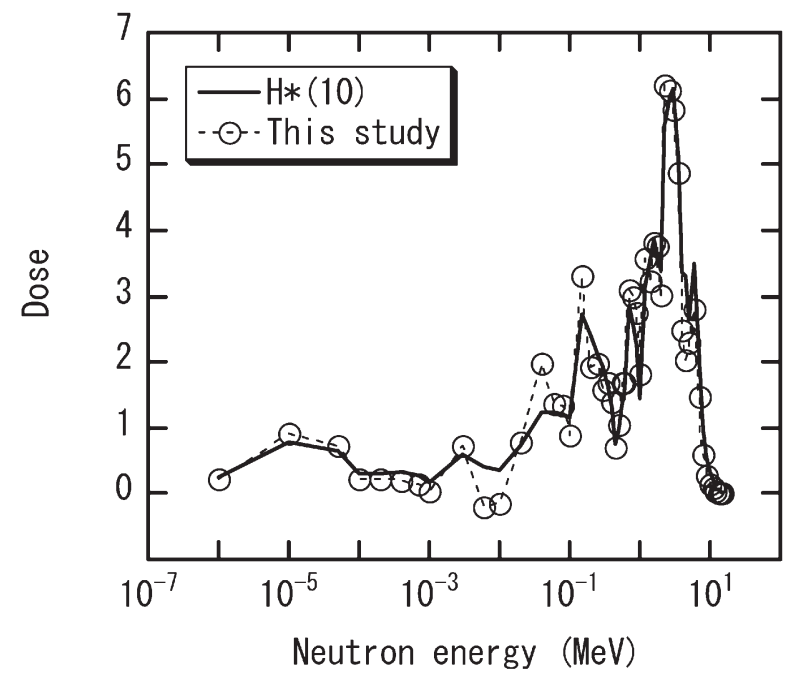

Fig. 2 Calculation result of the dose response to $\mathrm{Cf}-252\left(\mathrm{D}_{2} \mathrm{O}\right)$ moderated. 